\title{
Polarimetric radar signatures of precipitation at S- and C-bands
}

Prof. V.N. Bringi, PhD

V. Chandrasekar, PhD

P. Meischner, PhD

J. Hubbert, MS

Y. Golestani, PhD

\begin{abstract}
Polarimetric radar measurements in precipitation at $\mathrm{S}$ - and $\mathrm{C}$-band frequencies are considered. Time series data were obtained from three advanced radars: the National Center for Atmospheric Research (NCAR) CP-2 radar, the National Severe Storms Laboratory's (NSSL) Cimarron radar, and the C-band Poldirad radar operated by the German Aerospace Research Establishment (DLR). Measurements of radar reflectivity, differential reflectivity $Z_{D R}$, differential propagation phase $\phi_{D P}$, and the crosscorrelation between horizontal and vertical polarised waves are derived from time series data in rain, rain mixed with ice, and in the stratiform ice phase of convective storms. Raindrops are modelled as oblate in shape with a gamma form for their size distribution. The gamma parameters $\left(N_{o}, D_{o}, m\right)$ are varied over an entire range encompassing a wide variety of rainfall types. The radar rain measurements are shown to be in good general agreement with the model rain simulations. By combining $Z_{D R}$ and $\phi_{D P}$ it is possible to identify regions of mixed particle types, e.g. raindrops and hail, or ice crystals and snowflakes. The differential phase upon backscatter may be identified by examining the range profile of $\phi_{D P}$, giving additional clues as to the type and size of particles responsible for the backscatter.
\end{abstract}

\section{Introduction}

With the recent advent of sophisticated, polarimetricbased clutter characterisation methods, it is important to measure and model effectively the polarimetric back scatter and propagation effects of rainfall at typical microwave frequencies [1]. The raindrop size distribution (RSD) is governed by microphysical, thermodynamic and dynamical factors present in cloud systems. The RSD typically varies over orders of magnitudes both tempo-

Paper 7822F (E15), first received 3rd July 1989 and in final revised form Sth September 1990

Prof. Bringi, Mr. Hubbert and Dr. Golestani are with the Department of Electrical Engineering, Colorado State University, Fort Collins, CO 80523, USA

Dr. Chandrasekar is with the Department of Electrical Engineering University of Alabama in Huntsville, Huntsville, Alabama 35899, USA

Dr. Meischner is with the Institute of Atmospheric Physics, DLR NE-PA, Oberpfaffenhofen, D-8031 Wessling, Federal Republic of Germany

IEE PROCEEDINGS-F, Vol. 138, No. 2, APRIL 199I rally and spatially; for example, spatial variability is found in the interior of cumulo-nimbus clouds and the stratiform anvil regions of large storm systems. It is also well known that microwave/millimetre waves are significantly affected by rainfall, and the extent of attenuation, for example, suffered by electromagnetic waves depends on the RSD and its variability in space and time. The rain medium also introduces significant differential attentuation and differential phase shift between the principal polarisation states (namely, horizontal $(\mathbf{H})$ and vertical (V) polarisations) because the raindrops are oblate spheroidal in shape with a high degree of preferred orientation of their symmetry axis along the vertical direction. Additionally, the larger raindrops are uniformly more oblate than the smaller ones. A good review of these properties can be found in Reference 2 . While a substantial body of literature exists on the propagation aspects of microwave/millimetre waves through rainfaill, detailed modelling and experiments for different polarisation states is currently not available.

The use of advanced meteorological radars to characterise precipitation has reached a rather high level of sophistication only recently. The pioneering studies of McCormick, Hendry and their associates at the National Research Council of Canada have established the basis of polarimetric techniques, both for backscatter as well as forward scatter (propagation), using circular polarisation [3-6]. They have reported measurements of differential attenuaton and differential phase shift in rain at $\mathrm{K}-\mathrm{X}, \mathrm{X}$ and S-bands, using the range profile of $W / W_{2}$ were $W$ is the complex crosscovariance between the co- and crosspolar (simultaneously) received signals and $W_{2}$ is the conventional reflectivity. These studies have been extended [7-9] principally at S-band where differential phase shift is dominant with both absolute and differential attenuation being negligible.

The advent of pulse-to-pulse switching between $\mathrm{H}$ and $\mathrm{V}$ states and reception of copolar received signals through a single receiver system have advanced the accuracy to which the rain medium can be characterised [10, 11]. In additon, polarimetric techniques have found wide application in the detection of targets in clutter [12-14]. The technology of such polarimetric radar systems for meteorology has been reviewed by Bringi and Hendry [15], who also describe the main characteristics of 21 such radars existing worldwide. In this paper we present data from three advanced radars: the National Center for Atmospheric Research (NCAR) CP-2 radar located in Boulder, Colorado, USA, the National Severe Storm Laboratory's Cimarron radar located in Cimarron, Okla- 
homa, USA, and the C-band DLR radar located in Oberpfaffenhofen, Federal Republic of Germany. The main characteristics of the CP-2 and Cimarron radars are described in Reference 15 while the DLR radar is described in detail in Reference 16. Table 1, adapted from Reference 15 gives the salient features of these radar systems. where $\sigma_{H H}, \sigma_{V V}$ are the radar cross-sections at $\mathrm{H}$ and $\mathrm{V}$ states, respectively, and $N(D)$ is the particle size distribution. The wavelength is $\lambda$, while $K=\left(\varepsilon_{r}-1\right) /\left(\varepsilon_{r}+2\right), \varepsilon_{r}$ being the complex permittivity of the particle. The diffential reflectivity $Z_{D R}$ is defined as [10]

$$
Z_{D R}=10 \log \left(Z_{H H} / Z_{V V}\right) \mathrm{dB}
$$

\begin{tabular}{|c|c|c|c|}
\hline Characteristic & $\mathrm{CP}-2$ & Cimarron & Poldirad \\
\hline location & Boulder, CO, USA & $\begin{array}{l}\text { Cimarron, Oklahoma, } \\
\text { USA }\end{array}$ & $\begin{array}{l}\text { Oberpfaffenhofen, } \\
\text { Federal Republic of Germany }\end{array}$ \\
\hline wavelength, $\mathrm{cm}$ & 10.7 & 10.7 & 5.3 \\
\hline peak power, $\mathrm{kW}$ & 1200 & 500 & 500 \\
\hline pulse length, $\mu \mathrm{s}$ & 1 & 1 & $0.5,1.0,2.0$ \\
\hline $\begin{array}{l}\text { PRF, } \mathrm{s}^{-1} \\
\text { antenna type }\end{array}$ & $\begin{array}{l}\text { typically } 1000 \\
\text { centre-fed } \\
\text { paraboloid }\end{array}$ & $\begin{array}{l}1300 \\
\text { centre-fed } \\
\text { paraboloid }\end{array}$ & $\begin{array}{l}490,1200,2400 \\
\text { offset-fed } \\
\text { paraboloid }\end{array}$ \\
\hline beam width, deg. & 0.93 & 0.9 & 1 \\
\hline polarisations, radiated & $\mathrm{H}$ or $\mathrm{V}$ & $\mathrm{H}$ or $\mathrm{V}$ & $\begin{array}{l}\text { any elliptical, } 3^{\circ} \\
\text { increments in orientation } \\
\text { of ellipse }\end{array}$ \\
\hline polarisations, received & $\begin{array}{l}\text { copolar to } \\
\text { transmit } \\
\text { state }\end{array}$ & $\begin{array}{l}\text { copolar to } \\
\text { transmit } \\
\text { state }\end{array}$ & $\begin{array}{l}\text { copolar and crosspolar } \\
\text { to transmit } \\
\text { state }\end{array}$ \\
\hline $\begin{array}{l}\text { Doppler capability } \\
\text { range resolution, m }\end{array}$ & $\begin{array}{l}\text { yes } \\
\text { typically } 200\end{array}$ & $\begin{array}{l}\text { yes } \\
150\end{array}$ & $\begin{array}{l}\text { yes } \\
75,150,300\end{array}$ \\
\hline
\end{tabular}

The backscattered signals received by a radar have two primary features: $(a)$ parameters that are range cumulative, and $(b)$ variabilities that are functions of the backscatter properties of the medium. The rangecumulative features are functions of the foward-scatter properties of the raindrops whereas individual gate features are functions of the backscatter properties. These effects are not easily separable. Circular polarisation techniques have been used to separate out these effects, especially under propagation-dominated conditions [17]. In Reference 17 pulse-to-pulse switching between RHC and LHC states was not used, rather the transmitted pulse was RHC, the received wave being decomposed into its RHC and LHC components. Our techniques differ somewhat in that we employ pulse-to-pulse switching between $\mathbf{H}$ and $\mathrm{V}$ states (or orthogonal linear states) with coherent, copolar receptions through the same receiver and processor system.

This paper presents a study of several polarimetric observations of precipitation. The measurements analysed are as follows:

(a) radar reflectivity

(b) differential reflectivity between $\mathrm{H}$ and $\mathrm{V}$ polarisations

(c) differential propagation phase between $\mathrm{H}$ and $\mathrm{V}$ polarisations

(d) differential phase shift on backscatter and

(e) crosscorrelation between the $\mathrm{H}$ and $\mathrm{V}$ polarised signals.

\section{Polarimetric radar parameters}

Using a horizontal/vertical polarisation basis, we define the copolar radar reflectivities $Z_{H H}$ and $Z_{V V}$ as

$$
\begin{aligned}
Z_{H H} & =\frac{\lambda^{4}}{\pi^{5}|K|^{2}} \int \sigma_{H H}(D) N(D) d D \quad \mathrm{~mm}^{6} \mathrm{~m}^{-3} \\
Z_{V V} & =\frac{\lambda^{4}}{\pi^{5}|K|^{2}} \int \sigma_{V V}(D) N(D) d D \quad \mathrm{~mm}^{6} \mathrm{~m}^{-3}
\end{aligned}
$$

It is conventional to express the radar reflectivities in $\mathrm{dBZ}$ relative to $1 \mathrm{~mm}^{6} \mathrm{~m}^{-3}$, i.e. $10 \log \left(Z_{H H}\right)$.

If $S_{H H}, S_{V V}$ are the backscatter amplitudes at $\mathrm{H}$ and $\mathrm{V}$ states, then the crosscorrelation coefficient $\rho_{H V}$ can be defined as

$$
\rho_{H V}=\frac{\int S_{H H}(D) S_{V V}^{*}(D) N(D) d D}{\left\{\left[\int\left|S_{H H}\right|^{2} N(D) d D\right]\left[\int\left|S_{V V}\right|^{2} N(D) d D\right]\right\}^{1 / 2}}
$$

We further define $\rho_{H V}=\rho_{H V}(0) \exp (j \delta)$ where $\rho_{H V}(0)$ is the magnitude and $\delta$ the differential phase shift on backscatter. An explained later in Section 3, the ' 0 ' in brackets refers to zero time lag in radar systems using pulse-topulse switching between the transmitted $\mathrm{H}$ and $\mathrm{V}$ states.

If $f_{H H}(D)$ and $f_{V V}(D)$ are the forward-scatter amplitudes then the specific differential phase $K_{D P}$ is defined as

$$
K_{D P}=\frac{2 \pi}{k_{o}} \operatorname{Re} \int\left[f_{H H}(D)-f_{V V}(D)\right] N(D) d D \quad{ }^{\circ} \mathrm{km}^{-1}
$$

where $k_{o}$ is the free-space propagation constant. The two-way differential propagation phase between ranges $r_{1}$ and $r_{2}$ can be written as

$$
\phi_{D P}=2 \int_{r_{1}}^{r_{2}} K_{D P}(r) d r \text { degrees }
$$

Instead of choosing an $\mathrm{H} / \mathrm{V}$ basis, it is possible to choose a linear polarised basis, where the unit polarisation vectors are aligned at angles $\alpha$ and $\alpha+\pi / 2$ relative to the $\mathrm{H}$-direction. Thus, $Z_{D R}(\alpha)$ can be defined as the ratio (in decibels) between the copolar reflectivities at the two polarisation states defined by $\alpha$ and $\alpha+\pi / 2$. Similarly, we can define $\phi_{D P}(\alpha)$ and $\rho(\alpha)$ as the differential propagation phase and crosscorrelation coefficient, respectively, between the two polarisation states defined by $\alpha$ and $\alpha+\pi / 2$.

\subsection{Raindrops}

Microphysical properties of the rain medium that are important for radar observations are the raindrop size, shape and orientation distributions. The size distribution

IEE PROCEEDINGS-F, Vol I38, No. 2, APRIL I99! 
$N(D)$ may be modelled as a gamma distribution of the form [18]

$$
N(D)=N_{o} D^{m} \exp \left[-(3.67+m) D / D_{o}\right]
$$

$$
\mathrm{m}^{-3} \mathrm{~mm}^{-1}
$$

where $N(D)$ is the number of raindrops per unit volume per unit size interval $D$ to $D+\Delta D$. Note that $D$ refers to the equivalent spherical diameter, whereas $D_{o}$ is the median volume diameter. Drops larger than $D_{o}$ contribute to half the total rain-water content. Together with $N_{o}$ and $m$ the gamma distribution is characterised by three parameters whose physical variability is given in Reference 18, e.g. $N_{o}$ varies between 200 and $2 \times 10^{4}$ $\left(\mathrm{m}^{-3} \mathrm{~mm}^{-1-m}\right), D$ between 0.5 and $2.5 \mathrm{~mm}$, and $m$ between 0 and 5 . These parameter ranges reflect RSD variability found in differing storm types found worldwide [18].

The equilibrium shape of raindrops at terminal fall speed is that shape for which the forces due to surface tension, hydrostatic pressure and aerodynamic pressure (due to airflow around the drop) are in balance [19]. An approximation for the shape of large raindrops as oblate spheroidal with axis ratio (ratio of minor to major axis, $a / b)$ decreasing uniformly with increasing $D$ is given in Reference 20 . Recently, highly accurate computations of the shapes of large raindrops have been performed [21] In this paper, however, we use the simpler analytical solution for $a / b$ against $D$ (with $D$ in centimetres) derived in Reference 19:

$$
a / b=1.03-0.62 D
$$

The orientation of raindrops has been studied theoretically to show that the mean canting should be close to zero with standard deviation of the order of a few degrees [22]. These deductions are in good agreement with the observations of the canting-angle distributions of raindrops using linear and circular polarisation measurements [6]. In this paper we assume that the raindrops are equi-oriented with the minor axis of the oblate spheroid being vertical [23].

It is well known that in the Rayleigh limit $Z_{H H}$ is related to the sixth moment of the RSD, while $Z_{D R}$ is related to the reflectivity-weighted mean axis ratio of the raindrops filling the radar resolution volume. Because the mean axis ratio can be related to a mean size, $Z_{D R}$ is a monotonic function of the reflectivity-weighted mean drop size [24]. In Reference 36 it is shown from theoretical considerations that $\rho_{H V}(0)>0.99$ at $S$-band even in intense rain. In the Rayleigh limit, $\delta=0^{\circ}$; however, at higher frequencies (Mie region) $\delta$ can be quite significant. Note that $Z_{H H}, Z_{D R}, \rho_{H V}(0)$ and $\delta$ depend on the backscatter properties of the rain medium. At long wavelengths $K_{D P}$ is related to the mass-weighted (i.e. $D^{3}$ weighted) mean axis ratio of the raindrops [26]. Equivalently, $K_{D P}$ is nearly related to the $D^{4}$ th moment of the RSD since axis ratio and $D$ are related.

The raindrop model is now used to simulate the radar parameters defined in eqns. 1-4 at S- and C-bands. For each triplet of parameters $\left(N_{o}, D_{0}, m\right)$ we calculate the various radar observables using the previously defined shape and orientation assumptions. The parameters of the gamma RSD are then varied over the entire range prescribed in Reference 18 so that a scatter plot is generated, the scatter being representative of natural RSD fluctuations. Figs. 1-4 show the results of our simulations, the corresponding frequency band being denoted by $S$ or $C$ attached to the radar observables. Figs. $1 a$ and $b$ show

IEE PROCEEDINGS-F, Vol. 138, No. 2, APRIL 199I
$Z_{D R}$ against $Z_{H H}$ at $\mathrm{S}$ - and $\mathrm{C}$-band, respectively. At $Z_{H H}(S)=50 \mathrm{dBZ}, Z_{D R}(S)$ varies between 1.5 and $3 \mathrm{~dB}$, while at C-band $Z_{D R}(C)$ varies between 1.5 and $4 \mathrm{~dB}$ These $Z_{D R}$ variations at a fixed reflectivity level reflect the physical variability of the parameters $m$ and $D_{o}$ of the gamma RSD.
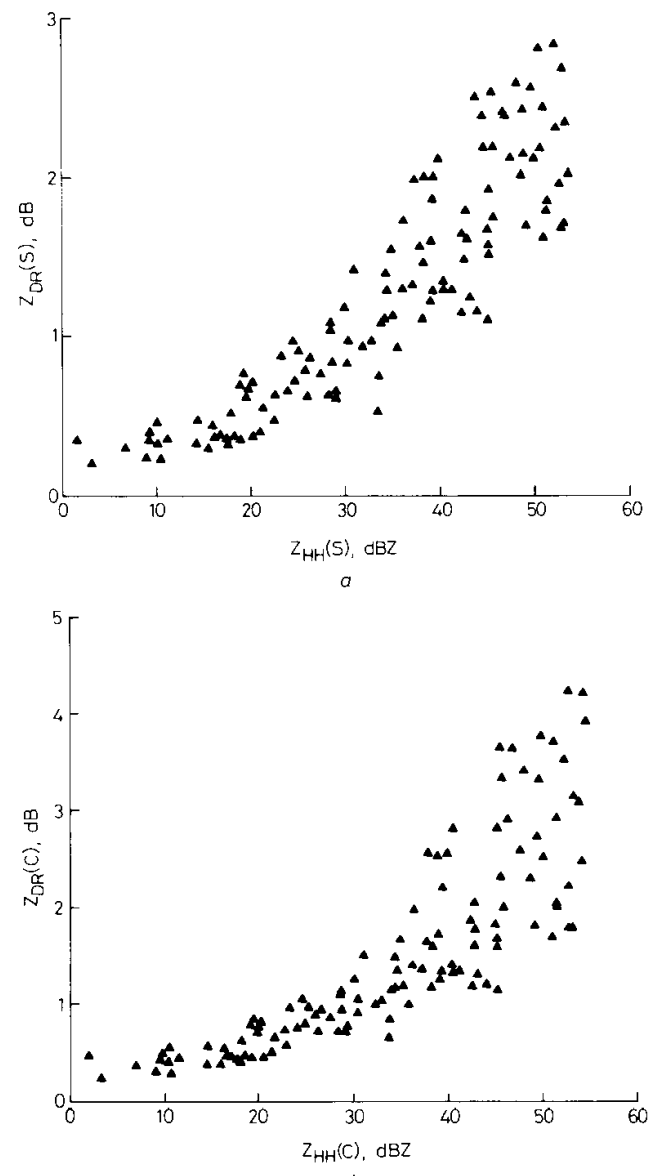

$b$

Fig. 1 Computations of $Z_{D R}$ against $Z_{H H}$ for oblate raindrops assuming a gamma size distribution

Each data point refers to a particular value of the triplet of gamma parameters $\left(N_{o}, D_{o}, m\right)$. The scatter of the data reflects a wide variety of rainfall types $a$ S-band $(10 \mathrm{GHz}) \quad b$ C-band $(5.5 \mathrm{GHz})$

Figs. $2 a$ and $b$ show $K_{D P}$ against $Z_{H H}$ at $\mathrm{S}$ - and C-band, respectively. At $Z_{H H}=50 \mathrm{dBZ}, K_{D P}(S)$ varies between 1.5 and $2{ }^{\circ} \mathrm{km}^{-1}$ whereas $K_{D P}(C)$ varies between 2 and $4 \mathrm{~km}^{-1}$. As is well known, this causes depolarisation of circularly polarised waves even at long wavelengths $[7,8]$. With intense rain rates and the possibility of long propagation paths, the use of circular polarisation for radars with only crosspolar reception (i.e. transmit $\mathrm{RHC}$, receive $\mathrm{LHC}$ ) must be avoided.

Fig. 3 shows $\rho_{H V}(0)$ against $Z_{H H}(S)$ and $\rho_{H V}(0)$ against $Z_{D R}(C)$. The various factors which contribute to $\rho_{u v}(0)$ deviating from unity are discussed in Reference 27. Principal among these are non-zero values of $\delta$ and/or mixed phase precipitation, i.e. rain mixed with partially melting ice or hail. At C-band large raindrops contribute substantially to $\delta$ as shown in Fig. 4 . 

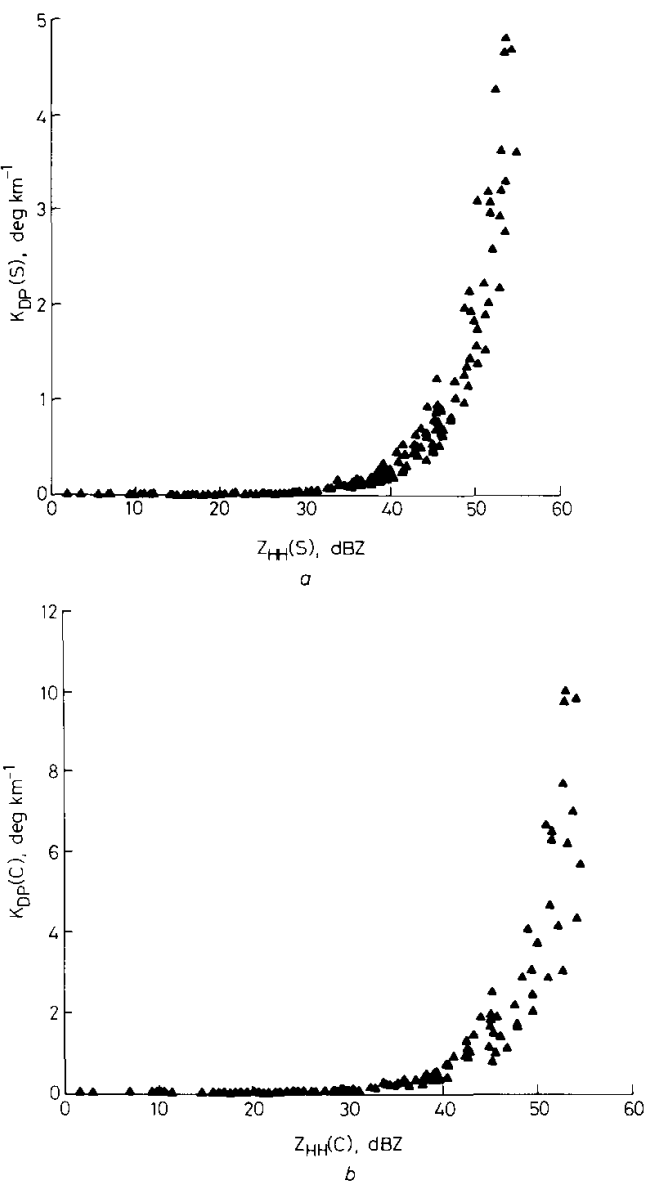

Fig. 2 Computations of $K_{D P}$ against $Z_{H H}$ for oblate raindrops assuming a gamma size distribution

Each data point refers to a particular value of the triplet of gamma parameters $\left(N_{o}, D_{o}, m\right)$. The scatter of the data reflects a wide variety of rainfall types

22 lce

The radar parameters defined earlier are dependent on the distributions of size, shape, orientation, and dielectric constant of the particles filling the radar resolution volume. For raindrops these distributions are well known but for a large variety of ice particles they are highly variable and thus assumptions must be made, particularly with respect to shape, orientation and composition of the ice particles. The melting of ice particles into raindrops has been modelled using a coupled radar-cum-melting model in References 28 and 29 , while in Reference 30 models for estimating rain and hail rates in mixed-phase precipitation using $Z_{H H}, Z_{D R}$, and $K_{D P}$ have been developed. The general shapes used for ice particles are either conical or spheroidal, the extreme oblate-prolate shapes being used for ice crystals (columns, needles or plates) The fall mode behaviour can be quite complex but often modelled as Gaussian or simple harmonic with the symmetry axes aligned in a mean sense along the vertical or horizontal directions. It is not the intent here to review the large amount of literature existing in the cloud physics or radar areas but we refer to the general Refer ences 2 and 31 .

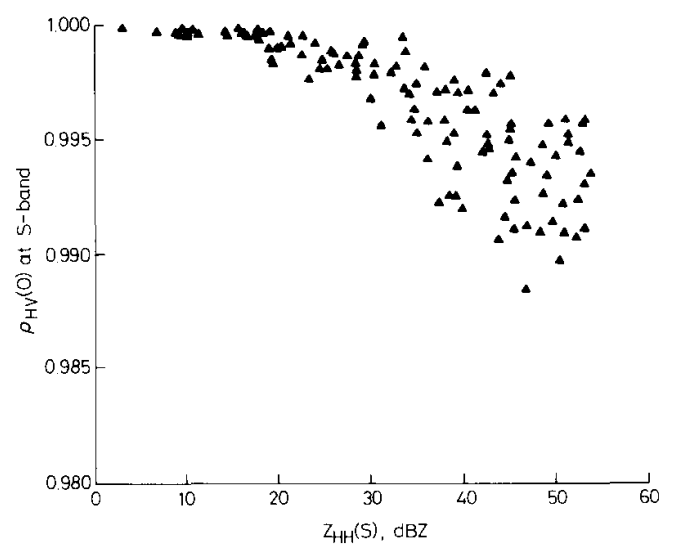

$a$

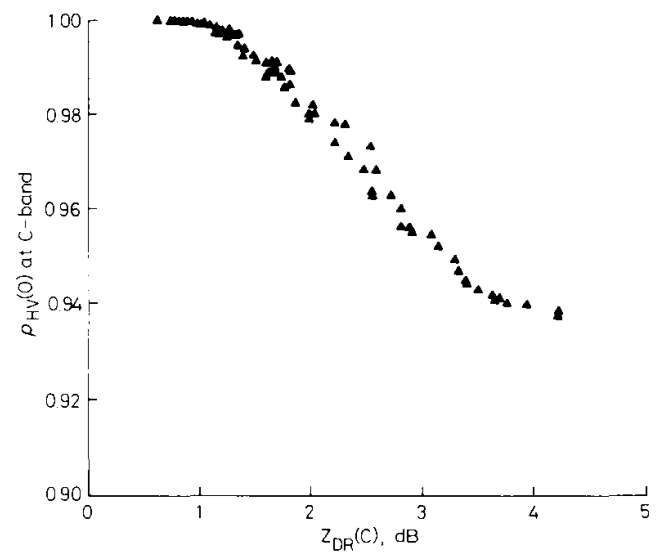

$b$

Fig. 3 Computations of $\rho_{H^{V}}(0)$ for oblate raindrops assuming a gamma size distribution

Each data point refers to a particular value of the triplet of gamma parameters $\left(N_{o}, D_{c}, m\right)$. The scatter of the data reflects a wide variety of rainfall types af $\rho_{H V}(0)$ against $Z_{H H}(S)$ at $S$-band
b $\rho_{H V}(0)$ against $Z_{D R}(C)$ at $\mathrm{C}$-band

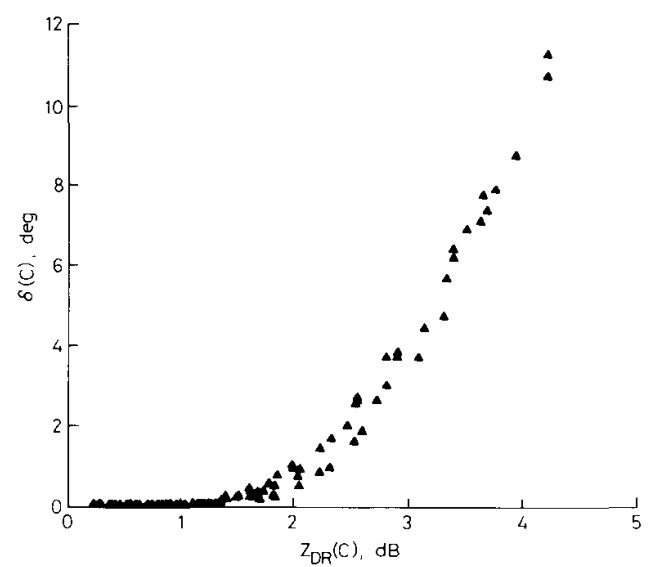

Fig. 4 Computations of $\delta$ against $Z_{D R}(C)$ for oblate raindrops assuming a gamma size distribution

Each data point refers to a particular value of the triplet of gamma parameters $\left(N_{\sigma}, D_{0}, m\right)$. The scatter of the datta reflects a wide variety of rainfall types

IEE PROCEEDINGS-F, VOI. 138, No. 2, APRIL I991 


\section{Polarimetric radar techniques}

Full polarimetric capability implies polarisation diversity on transmission and reception [12]. The NCAR CP-2 radar is a dual-frequency ( $\mathrm{S}-/ \mathrm{X}$-bands) radar which can measure $Z_{D R}$ and $\phi_{D P}$ at $\mathrm{S}$-band using pulse-to-pulse switching of the transmitted polarisation between $\mathrm{H}$ and $\mathrm{V}$ states. In addition, the ratio of reflectivities at $\mathrm{S}$ - and $\mathrm{X}$-bands can be measured. The Cimarron radar operated by the National Severe Storms Laboratory (NSSL) is also equipped for $Z_{D R}$ and $\phi_{D P}$ at $S$-band using pulse-topulse switching. The C-band Poldirad radar operated by the German Aerospace Research Establishment (DLR) possesses full polarimetric capability, i.e. pulse-to-pulse switching between orthogonal states with corresponding dual-channel reception $[16,23]$. Unless specified otherwise, we utilise only a subset of this measurement capability, i.e. switching between $\mathrm{H}$ and $\mathrm{V}$ states with copolar reception through the same receiver and processor. A brief description of these 3 radars is provided in Table 1 (see also Reference 15).

The measurement of $Z_{D R}$ and $\phi_{D P}$ as well as the firs three moments of the Doppler spectrum when a pulsed Doppler radar is used for transmitting alternately polarized $\mathrm{H}$ and $\mathrm{V}$ pulses, with coherent reception of the copolar signals through the same receiver system, is discussed by Sachidananda and Zrnic [32]. Their algorithm for $\phi_{D P}$ is based on Reference 33. If $H_{2 n}, V_{2 n+1}$ represent the sample voltages at a fixed range delay $r$ due to $\mathrm{H}$ and $V$ transmitted pulses, respectively, then $Z_{D R}$ can be estimated from the mean sample powers $\hat{P}_{H H}$ and $\hat{P}_{V V}$, where

$$
\begin{aligned}
& \hat{P}_{H H}=\frac{1}{M} \sum_{i=0}^{M-1}\left|H_{2 i}\right|^{2} \\
& \hat{P}_{V V}=\frac{1}{M} \sum_{i=0}^{M-1}\left|V_{2 i+1}\right|^{2} \\
& \hat{Z}_{D R}=10 \log \left(\hat{P}_{H H} / \hat{P}_{V V}\right)
\end{aligned}
$$

and $M$ is the number of $\mathrm{H} / \mathrm{V}$ sample pairs

The estimate for $\phi_{D P}$ from Reference 33 is

$$
\hat{\phi}_{D P}=\frac{1}{2} \arg \left(\hat{R}_{a} \hat{R}_{b}^{*}\right)
$$

where

$$
\begin{aligned}
& \hat{R}_{a}\left(T_{S}\right)=\frac{1}{M} \sum_{i=0}^{M-1} H_{2 i}^{*} V_{2 i+1} \\
& \hat{R}_{b}\left(T_{S}\right)=\frac{1}{M} \sum_{i=0}^{M-1} V_{2 i+1}^{*} H_{2 i+2}
\end{aligned}
$$

$T_{S}$ being the pulse repetition time.

$\hat{\phi}_{D P}$ at two ranges $r_{1}$ and $r_{2}$ can be used to calculate the specific differential phase shift as

$$
K_{D P}=\frac{\hat{\phi}_{D P}\left(r_{2}\right)-\hat{\phi}_{D P}\left(r_{1}\right)}{2\left(r_{2}-r_{1}\right)}
$$

The crosscorrelation coefficient $\hat{\rho}_{H V}(0)$ at zero time lag is obtained from Reference 32 .

$$
\hat{\rho}_{H V}(0)=\left|\hat{\rho}_{H V}\left(T_{S}\right) / \hat{\rho}\left(T_{S}\right)\right|
$$

where

$$
\hat{\rho}_{H V}\left(T_{S}\right)=\frac{\left|\hat{R}_{a}\left(T_{S}\right)\right|+\left|\hat{R}_{b}\left(T_{S}\right)\right|}{2\left(\hat{P}_{H H} \hat{P}_{V V}\right)^{1 / 2}}
$$

IEE PROCEEDINGS-F, Vol. 138, No. 2, APRIL I99/ and $\hat{\rho}\left(T_{S}\right)$ is the autocorrelation estimate of the $\mathrm{H}$ polarised signals. This estimate is obtained by assuming a Gaussian Doppler spectrum for which $\left|\rho\left(T_{S}\right)\right|=$ $\left|\rho\left(2 T_{S}\right)\right|^{1 / 4}$. Note that $\hat{\rho}\left(2 T_{S}\right)$ is obtained directly from the $H$ - or $V$-polarised signals.

To meaningfully compare radar data with model computations, the statistical fluctuations in the radar measurements must be reduced to a level smaller than the physical variabilities. Statistical fluctuations have been studied for the first three moments of the Doppler spectrum [34]. The standard errors quoted assume that the Doppler spectrum width is in the range $1-4 \mathrm{~ms}^{-1}$, that the number of sample pairs is $100-200$, that the mean $\rho_{H V}(0)$ is $0.99-0.995$ and that SNR $>20 \mathrm{~dB}$. The zeroth moment corresponding to the conventional reflectivity can be estimated with a standard error of $1 \mathrm{~dB} . \hat{Z}_{D R}$ can typically be estimated to within $0.1-0.2 \mathrm{~dB}[35,36]$. $\hat{\phi}_{D P}$ errors have been studied by Sachidananda and Zrnic [25], who show theoretically that the standard error in $\hat{\phi}_{D P}$ is the order of a few degrees. The errors in $\hat{Z}_{D R}, \hat{\phi}_{D P}$ and $\hat{\rho}_{H V}(0)$ are very sensitive to SNR and the mean value of $\rho_{H V}(0)$. These standard errors are applicable to data from a single resolution volume, with further reduction made possible by averaging (filtering) contiguous range gates.

The estimation of $K_{D P}$ is based on the range profile of $\phi_{D P}$. A third-order nondecreasing polynomial fit to the $\phi_{D P}$ range profile is one method used to estimate $K_{D P}$ at each resolution volume. We also perform range filtering on the various radar observables based on infinite impulse response (IIR) digital filters. To estimate $K_{D p}$, for example, the range profile of $\phi_{D P}$ would be digitally filtered to suppress the 'high frequency' range gate-to-range fluctuations while still preserving the mean trend with range. This procedure significantly decreases the standard error in $K_{D^{p}}$ to typically around $0.4^{\circ} \mathrm{km}^{-1}$

\section{$4 \quad$ Radar measurements}

\subsection{CP-2 radar}

We first present radar data obtained with the NCAR CP2 radar which was located near Boulder, Colorado, USA during the summer of 1987 . The radar was operated in a special time series mode for brief periods of time. The transmitted wave's polarisation state was switched between $\mathrm{H}$ and $\mathrm{V}$ states on alternate pulses while the received signal was always copolar to that transmitted. Range profiles of $Z_{H H}, Z_{D R}, \phi_{D P}$ and $\rho_{H V}(0)$ were generated using techniques described in Section 3. Figs. $5 a$ and $b$ show a typical range profile through an intense convective storm at low elevation angle. In the range interval 92 to $98 \mathrm{~km}$ the following can be deduced, (i) average $K_{D P}=2.8^{\circ} \mathrm{km}^{-1}$, (ii) $Z_{H H}(S)>55 \mathrm{dBZ}$, (iii) average $Z_{D R} \simeq 1.5 \mathrm{~dB}$ and (iv) $\rho_{H V}(0) \lesssim 0.90$. From Fig. $1 a$ we can ascertain that for $Z_{H}=55 \mathrm{dBZ}$ the corresponding $Z_{D R}$ for a pure rain medium would be in the range $1.75-3.00 \mathrm{~dB}$ while $\rho_{H V}(0)>0.99$. Thus, in the example at hand we can infer that mixed phase precipitation is occurring in the range interval $92-98 \mathrm{~km}$, most probably intense rain mixed with hail. Using the relation $R=40.5\left(K_{D P}\right)^{0.85}$ gives a rain rate of $97 \mathrm{~mm} \mathrm{hr}^{-1}$ with $K_{p p}(S)=2.8^{\circ} \mathrm{km}^{-1}$ [37]. To derive the rain rate we assume that the hail particles are quasi-spherical and tumble giving $f_{H H} \simeq f_{V V}$ [30]. Thus, they will not contribute to $K_{D P}$ whereas the raindrops will (see Reference 4).

We accumulated a large number of range profiles through convective rainshafts from several different storms during the summer of 1987. Care was taken to 
exclude mixed-phase precipitation by eliminating data with low values of $\rho_{H V}(0)$ or where the dual-frequency (Sto $\mathrm{X}$-band) reflectivity ratio (or hail signal) exceeded
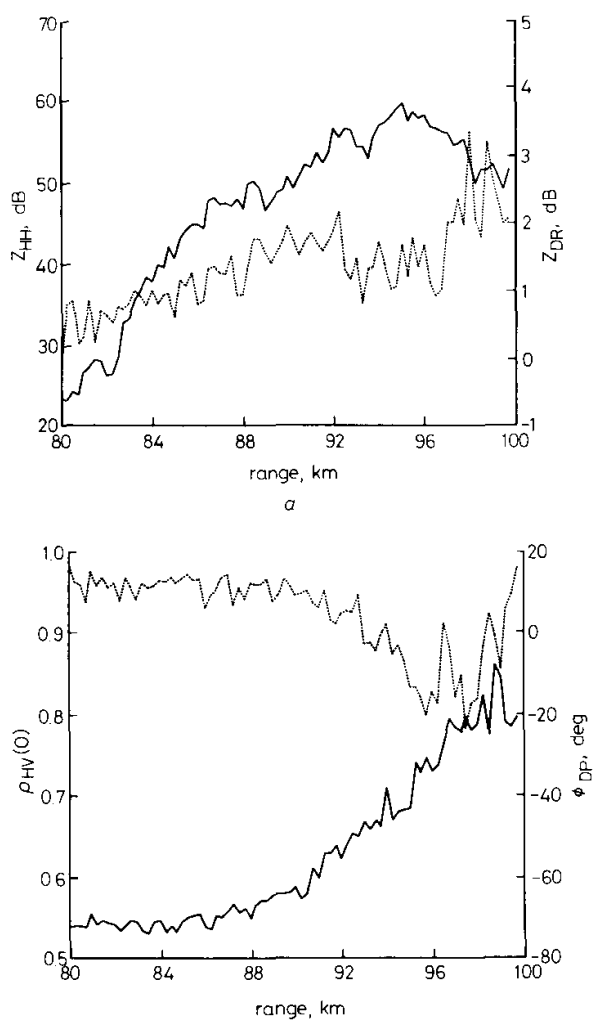

$b$

Fig. 5 Range profile of radar measurements obtained using the NCAR $C P-2$ radar at low elevation angle through a convective storm

29 th July $1987 ; 20: 38 ;$ azimuth $=125 ;$ elevation $=1.0$

a $Z_{H H}$ and $Z_{D R} ;-$ reflectivity, $\cdots \cdots$ differential reflectivity

$b \phi_{D P}$ and $\rho_{H V}(0) ; \cdots-$ propagation differential phase, $\cdots \cdots$ zero-lag crosscorrelation

$3 \mathrm{~dB}$. The $Z_{H H}$ and $Z_{D R}$ fields were filtered in range using a weighted, moving-average filter. A scatterplot of $Z_{D R}(S)$ against $Z_{H H}(S)$ was constructed from this fairly large database and is shown in Fig. 6a. This plot can be compared with Fig. 1a. For convenience, the two dashed curves shown in Fig. $6 a$ were obtained from Fig. $1 a$ as the upper and lower bounds in $Z_{D R}$ for a given $Z_{H H}$. It is clear that the measurements fall within the bounds based on RSD fluctuations.

Fig. $6 b$ shows the average value of $\rho_{H V}(0)$ against $Z_{H H}(S)$, the average being done over $1 \mathrm{~dB}$ categories of $Z_{H H}$. The database is the same as that used in Fig. $6 a$. The vertical bars denote the standard deviation. The dashed curve is the mean relationship drawn by eye from Fig. $3 a$. The radar measurements are thus seen to be in good agreement with the RSD simulations. Note that the RSD simulations of $\rho_{H V}(0)$ account for variable raindrop size distributions as well as the mean relationship between axis ratio and size, see eqns. 6 and 7 .

Fig. 7 shows range profiles of (a) $Z_{H H}(S)$, (b) $Z_{D R}(S)$ and $(c) \phi_{D P}(S)$ through the ice phase of a convective storm at high elevation angle. The convective core is located in the range interval $20-40 \mathrm{~km}$, i.e. high reflectivity. Beyond $40 \mathrm{~km}$ the profile penetrates through the (low reflectivity) stratiform anvil region of the storm. An
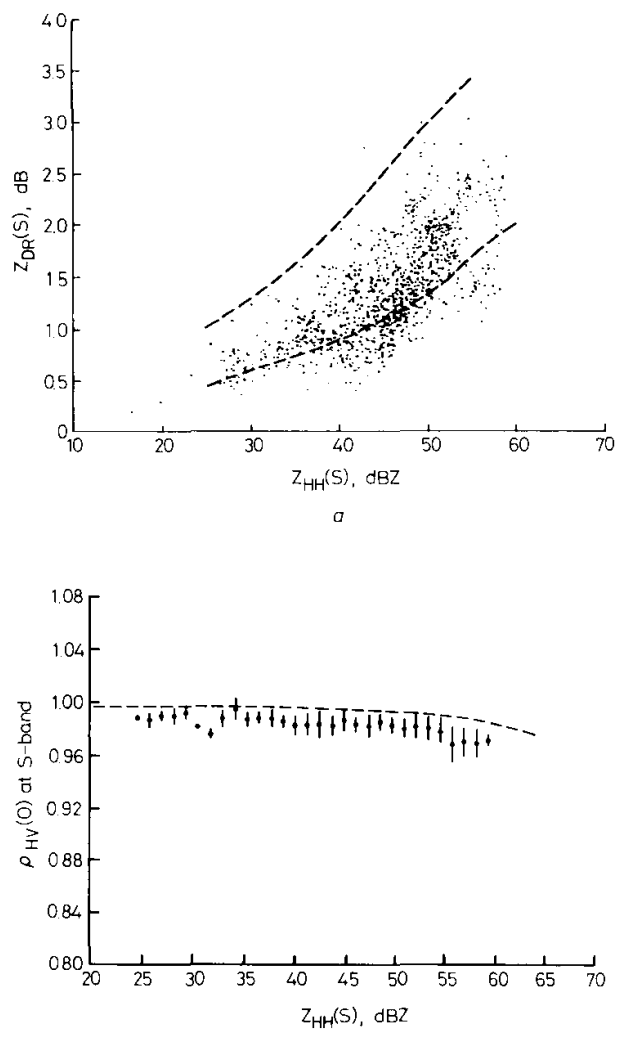

Fig. $6 Z_{D R}(S)$ and $\rho_{H V}(0)$ for data taken in convective rainfall using the CP-2 radar

a Scatterplot of radar measured $Z_{D R}(S)$ against $Z_{H H}(S)$. Dashed lines are drawn in as the upper and lower bounds from the data of Fig. $1 a$

b. Radar measured $\rho_{H V}(0)$ against $Z_{H H}(S)$. The averaging is done over $I \mathrm{~dB}$ categories of $Z_{H H}$. Vertical lines represent the standard deviation. The dashed line is drawn in as the mean fit (by eye) to the calculations shown in Fig. $3 a$

average $K_{D P}$ of $0.6^{\circ} \mathrm{km}^{-1}$ is estimated in the stratiform region $(40-60 \mathrm{~km})$. Oriented ice crystals of the plate type can give such large $K_{D P}$ values [17]. Because $Z_{D P}$ is low in this region $(0-0.5 \mathrm{~dB})$ we hypothesize that larger snowflakes which are quasi-spherical and tumble are mixed in with the oriented crystals. Again, they do not contribute to $K_{D P}$ because these particles have $f_{H H} \simeq f_{V V}$, while the oriented plate-type crystals do contribute to $K_{D P}$ via $f_{H H}$ $-f_{V V}$ in eqn. 4. Since $Z_{D R}$ is a measure of the reflectivityweighted mean shape of the mixed-phase precipitation, it is biased towards $0 \mathrm{~dB}$ by the larger, quasi-spherical snowflakes whose $\sigma_{H I J} \simeq \sigma_{V V}$.

\subsection{Cimarron Radar}

We now present radar measurements using the NSSL S-band Cimarron radar located NW of Oklahoma City, Oklahoma, USA. This radar was operated in a special time series mode for brief periods of time during the Spring of 1989. Data from this radar have been reported earlier by $[25,30]$. A high-power polarisation switch was used to transmit alternately $\mathrm{H}$ - and $\mathrm{V}$ polarised pulses, the received signal being always copolar 
to that transmitted. Thus, range profiles similar to Fig. 5 could be generated from the time series data. Figs. 8 and 9 show examples through an intense convective storm at
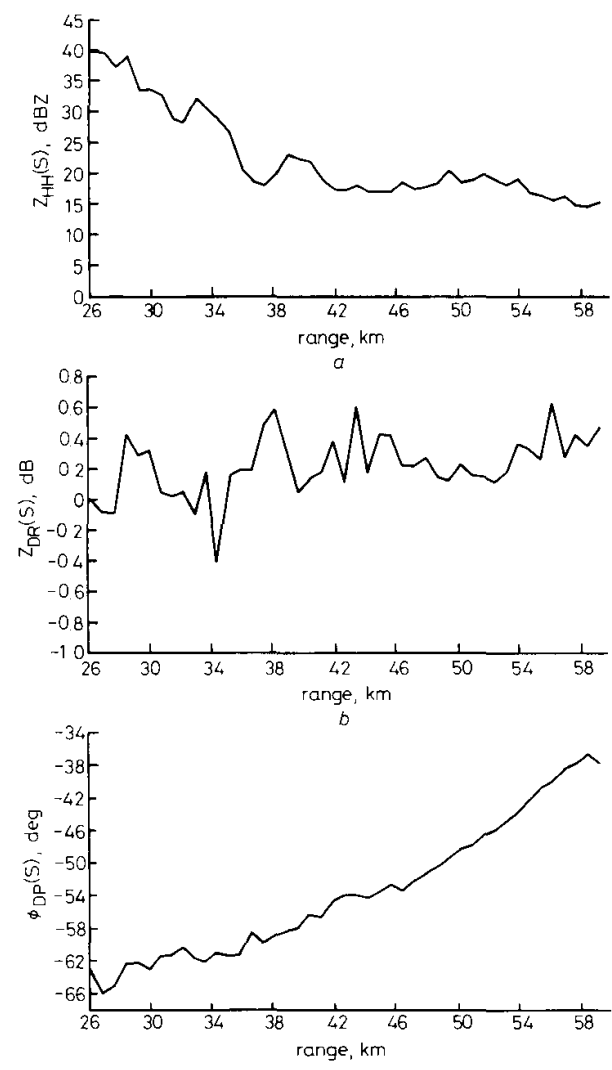

Fig. 7 Range profile of $\mathrm{CP}-2$ radar measurements taken at high elevation angle through the ice phase of convective and stratiform parts of a storm

The convective core is located between ranges 20 and $40 \mathrm{~km}$. The stratiform part extends beyond $40 \mathrm{~km}$.

a $Z_{\mathrm{HH}}(S)$

$c \phi_{D P}(S)$

a low elevation angle of $0.5^{\circ}$. Fig. $8 a$ shows $Z_{H H}$ and $Z_{D R}$ against range while Fig. $8 b$ shows $\phi_{D P}$ and $\rho_{H Y}(0)$ against range. The sharp increase in $\phi_{D P}$ in the range $38-50 \mathrm{~km}$ is noteworthy. In the range interval $42-49 \mathrm{~km}, Z_{D R}$ is in the range $1-1.5 \mathrm{~dB}$, while $Z_{H H}$ is in the range $55-60 \mathrm{dBZ}$. Thus, this case is similar to Fig. 5 in that intense rain mixed with hail is occurring in the range $42-49 \mathrm{~km}$. Fig. 9 shows the IIR filtered version of $\phi_{D P}$. This filters out the 'high frequency' gate-to-gate variations while still preserving the mean trend with range. Fig. 9 also shows the mean Doppler velocity against range. The mean Doppler velocity is obtained by combining eqns. $10 a$ and $10 b$ as suggested by Sachidananda and Zrnic [32]. In the intense precipitation shaft $(42-49 \mathrm{~km})$ the $K_{D P}$ peaks to around $4 \mathrm{~km}^{-1}$ or a rain rate of $132 \mathrm{~mm} \mathrm{hr}^{-1}$. The mean radial velocity profile shows a sharp transition from $-12 \mathrm{~ms}^{-1}$ at $43 \mathrm{~km}$ to $+12 \mathrm{~ms}^{-1}$ at $49 \mathrm{~km}$. Negative (positive) radial velocities represent winds toward (away from) the radar. Thus, the intense precipitation at the surface coincides with a region of strong radial diver-

IEE PROCEEDINGS-F, Vol. 138, No. 2, APRIL I99I gence of the wind. This signature is referred to as a microburst which causes damaging winds at the surface.

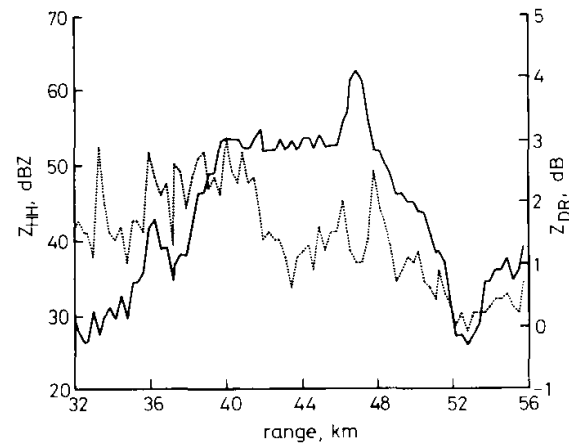

$a$

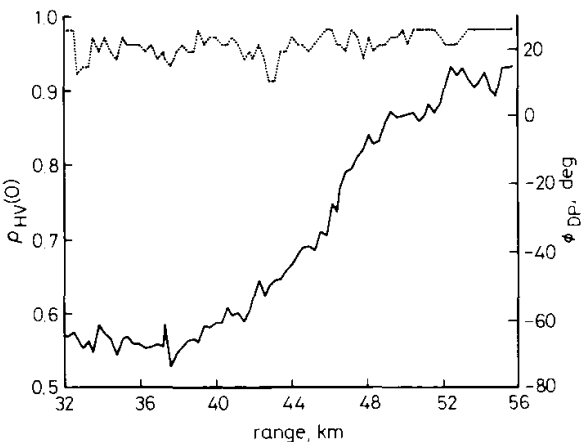

$b$

Fig. 8 Range profile at $S$-band of Cimarron radar measurements taken at low elevation angle through an intense storm

6th June $1989 ; 14: 34: 31 ;$ azimuth $=285 ;$ elevation $=0.5$

$a Z_{H H}$ and $Z_{D R} ;-$ reflectivity, $\cdots \cdots$ differential reflectivity $b \phi_{D P}$ and $\rho_{H V}(0) ;-\quad$ propagation differential phase, $\cdots \cdots$ zero-lag crosscorrelation

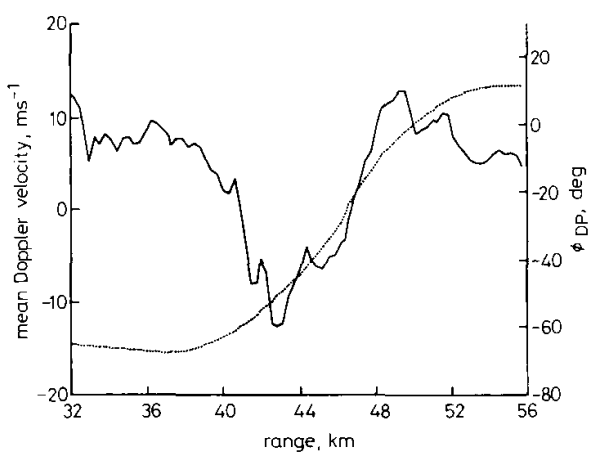

Fig. 9 An IIR digital filter is applied to the $\phi_{D P}$ data of Fig. $8 b$ to filter out the 'high frequency' range gate-to-range gate variations, while still retaining the mean increasing trend with range. Also shown is the mean Doppler velocity profile corresponding to a downburst case

$$
\text { mean Doppler velocity, } \cdots \cdots \text { IIR filtered phase }
$$

The precipitation shaft is colocated with a strong, vertically directed downdraft which diverges radially near the surface [38].

Fig. 10 shows an example of a range profile through the stratiform anvil region of a mesoscale convective complex. The reflectivity 'bright band' is located near 33$35 \mathrm{~km}$ where a peak in $Z_{D R}$ of $1.2 \mathrm{~dB}$ is noted. Beyond 
the 'bright band' the reflectivity falls off rapidly while at the same time $\phi_{D P}$ shows a monotonic increasing trend with range, see Fig. $10 b$. At the 'bright band' $\rho_{H V}(0)$

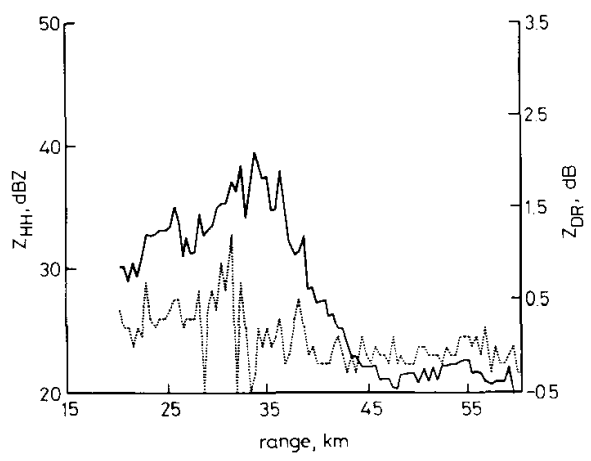

$a$

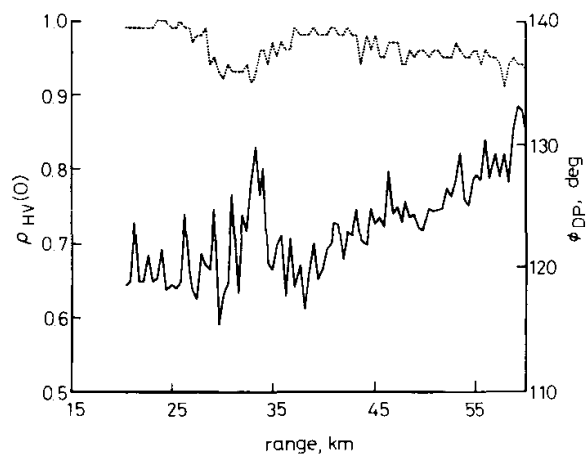

Fig. 10 Range profile of Cimarron radar measurements through the bright band' of a stratiform anvil region of a mesoscale convective system 3rd June 1989;21:08; azimuth $=28$; elevation $=4.9$ The "bright band" is located in the range $33-35 \mathrm{~km}$

a $Z_{H H}$ and $Z_{D R} ;-\quad$ reflectivity, $\cdots \cdots$ differential refectivity $b \phi_{D P}$ and $\rho_{P V}(0)$. Note the minima in $\rho_{U V}(0)$ at the 'bright band'. - propgation differential phase. ........ zero-lag crosscorrelation

shows a minima of around 0.9. Significant deviation of $\rho_{H V}(0)$ from unity occurs in the interval $29-33 \mathrm{~km}$. In this same region $\phi_{D P}$ fluctuations are larger and near $33 \mathrm{~km} \mathrm{a}$ 'bump' in $\phi_{D P}$ above the mean trend is noticed. If $\delta=0^{\circ}$, then eqn. 9 gives the true differential propagation phase. However, if $\delta$ is non-zero then eqn. 9 gives the profile of $\left(\phi_{D P}+\delta\right)$, where $\phi_{D P}$ is the range cumulative part and $\delta$ is the backscatter differential phase at a particular range location. In practice, it is difficult to separate out the two components. In this particular example, a mean $\phi_{D P}$ trend with range can be identified and a peak $\delta \simeq 10^{\circ}$ may be estimated in the 'bright band'. The 'bright band' is known to consist typically of large, melting snowflakes, ice crystals and small raindrops. We believe that nonzero $\delta$ causes the sharp reduction of $\rho_{H V}(0)$ near the "bright band' (see also Reference 39). In the range interval 40 $60 \mathrm{~km}$, a mean $K_{D P} \simeq 0.25^{\circ} \mathrm{km}^{-1}$ may be deduced. As discussed earlier in reference to Fig. 7, we infer that this $K_{p p}$ is caused by oriented, plate-type ice crystals mixed with larger, quasi-spherical snowllakes. Mixed-phase precipitation is inferred since $Z_{D R} \simeq 0 \mathrm{~dB}$ in this range interval. The decrease in $\rho_{H Y}(0)$ with range beyond $45 \mathrm{~km}$ is most probably due to low signal-to-noise ratio.

\subsection{Poldirad}

The German Aerospace Research Establishment (DLR) operates an advanced $\mathrm{C}$-band polarimetric radar called Poldirad. This radar is located near Munich, Germany. A good technical description of the radar and examples of data taken in convective and stratiform conditions are given in Reference 16.

In the fast-switching time series mode, the polarisation state of the transmitted wave is switched alternately between any two selected orthogonal states on a pulseto-pulse basis. We have selected the $\mathrm{H} / \mathrm{V}$ states. For each transmit pulse, the inphase and quadrature-phase components of the copolar and crosspolar receive signals are recorded. Only the copolar time series data are used here to estimate $\phi_{D P}$ and $\rho_{H V}(0)$ as in eqns. 9 and 12 . Fig. 11 shows an example of a range profile through a convective cell at an elevation angle of $2.1^{\circ}$. These data were gath ered on 23rd August, 1988. Fig. 11a shows the reflectivity and $Z_{D R}$ profile, and Fig. $11 b$ shows the profiles of $\phi_{D P}$ and $\rho_{H V}(0)$. Note the steady increase of $\phi_{D P}$ with range characteristic of propagation differential phase in rainfall. $Z_{D R}$ typically lies in the range $0-3.5 \mathrm{~dB}$. The standard error of $\phi_{D P}$ was estimated from the time series data to be in the range $2-4^{\circ}$ when $\rho_{H V}(0)>0.95$ and 64 pairs of $\mathrm{H} / \mathrm{V}$ samples are used, i.e. $M=64$ in eqns. 9 and 10 . Fig. $11 b$ shows an IIR filter applied to the $\phi_{D P}$ data which is shown by the dashed line which effectively filters out the high-frequency range gate-to-range gate variations, yet preserving the mean trend with range. $K_{D P}$ values determined from the filtered $\phi_{D P}$ data were in the range 0 $2^{\circ} \mathrm{km}^{-1}$. These radar measurements can be compared to the C-band rainfall simulations shown in Figs. 1-4.
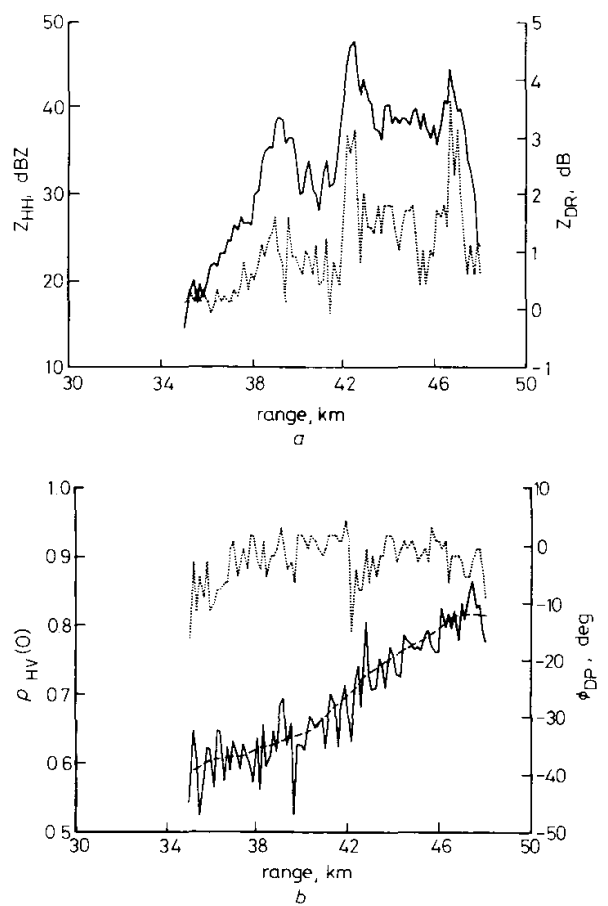

Fig. 11 Range profiles of Poldirad measurements through a convective rainshaft at low elevation angle of $2.1^{\circ}$

23 rd August 1988; $16: 23 ;$ azimuth $=120 ;$ elevation $=2.1$ a $Z_{H H}$ and $Z_{D R} ;-$ reflectivity, ...... differential reflectivity $b \phi_{D P}$ and $\rho_{H V}(0) ; \frac{\text { propagation differential phase, } \cdots \cdots \text { zero-lag cross }}{\text { correlation, }---- \text { IIR filtered } \phi_{D P}}$
con

IEE PROCEEDINGS-F, Vol. 138, No. 2, APRIL 199 
A number of range profiles (acquired over $1 \mathrm{~min}$ ) similar to Fig. 11 were analysed with IIR filtering applied additionally to $Z_{H H}$ and $Z_{D R}$. This filter was designed to suppress random measurement errors, yet the range gateto-range gate variability was retained. Fig. 12 shows a scatterplot of $Z_{D R}$ against $Z_{H H}$ which may be compared to the simulations of Fig. 1b. The two dashed lines in Fig. 12 were obtained from Fig. $1 b$. They represent the upper and lower bounds in $Z_{D R}$ for a given $Z_{H H}$. The radar measurements in rainfall are thus seen to fall within the bounds established by the simulations.

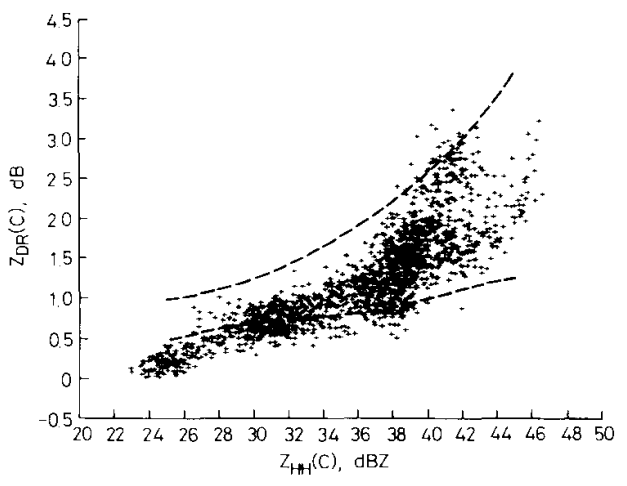

Fig. 12 Scatterplot based on 20 range profiles similar to Fig. 11 of radar measured $Z_{D R}(C)$ against $Z_{H H}(C)$ taken in convective rainfall using Poldirad

Dashed lines are drawn in as the upper and lower bounds from the data of Fig $1 \mathrm{~b}$ 23 rd August 1988, time $-16: 23 \cdot 30-16: 24: 14$

We now consider the ray profile taken through another isolated convective cell on 23rd August, 1988 with higher $Z_{H H}$ values. This case is described in some detail in Reference 23. Fig. 13 shows the ray profile: Fig. $13 a$ shows $Z_{H H}$ and $Z_{D R}$ and Fig. $13 b$ shows $\phi_{D P}$ and $\rho_{H V}(0)$. Note the very high $Z_{D R}$ in the range $57-58 \mathrm{~km}$, as well as the relatively low $\rho_{H V}(0)$ values. The $\phi_{D P}$ profile consists of 'bumps' superposed on a monotonic increasing trend; these 'bumps' are possibly due to differential phase shift on backscatter $(\delta)$. The IIR filtered version of $\phi_{D P}$ is shown by the dashed line in Fig. $13 b$, the filter being designed to preserve the 'bumps' rather than the mean trend with range. Note that the $\phi_{D P}$ "bumps' centred at ranges $58,60.5$ and $62.75 \mathrm{~km}$ are correlated with $Z_{D R}$ peaks of 6,4 and $3 \mathrm{~dB}$, respectively, and possibly with local minima in $\rho_{H V}(0)$. In the range interval

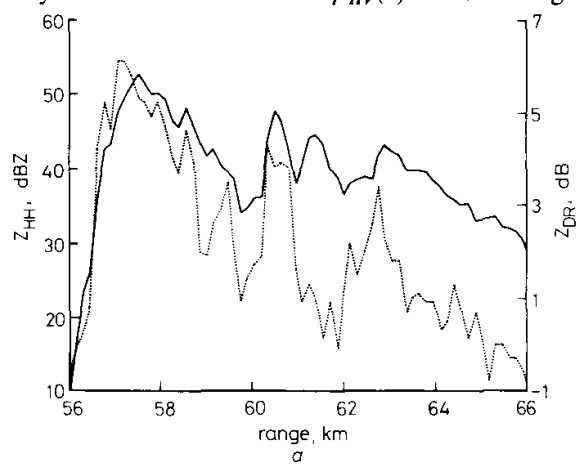

Fig. 13 Range profiles of Poldirad measurements taken through a convective storm

23 rd August 1988; $15: 20 ;$ azimuth $=115$; elevation $=1.65$

a $Z_{H H}$ and $Z_{D R}:-$ reflectivity, $\cdots \cdots$ differential reflectivity

$b$ and $\rho_{W V}(0) ; \ldots$ - propagation differential phasc. ….... zero-lag cross-correlation, . . - IIR filtered $\phi_{D A}$

The filter is designed to preserve the 'bumps' due to backscatter differential phase in the raw $\phi_{D P}$ range profile

IEE PROCEEDINGS-F, Vol. 138. No. 2, APRIL I99]
$57-58.5 \mathrm{~km}$ we can estimate $\delta$ to be in the range $13-18^{\circ}$, $Z_{D R}$ in the range $4-6 \mathrm{~dB}$, and reflectivity $45-55 \mathrm{dBZ}$. The significantly low values of $\rho_{H V}(0)$ imply that rain mixed with ice (possibly small, melting hailstones) is occurring in the range interval $57-58.5 \mathrm{~km}$. The steady decrease in $Z_{D R}$ with range beyond $58 \mathrm{~km}$ (except for local peaks at 60.5 and $63 \mathrm{~km}$ ) is caused by differential attenuation. This ray profile shows the difficulty of separating out backscatter effects due to $\delta$ from forward-scatter effects, i.e. estimating $K_{D P}$, using just the $\phi_{D P}$ range profile.

Fig. $14 a$ shows $Z_{D R}$ against $Z_{H B}$ obtained from 20 profiles (over a 1 min time interval) similar to Fig. 13
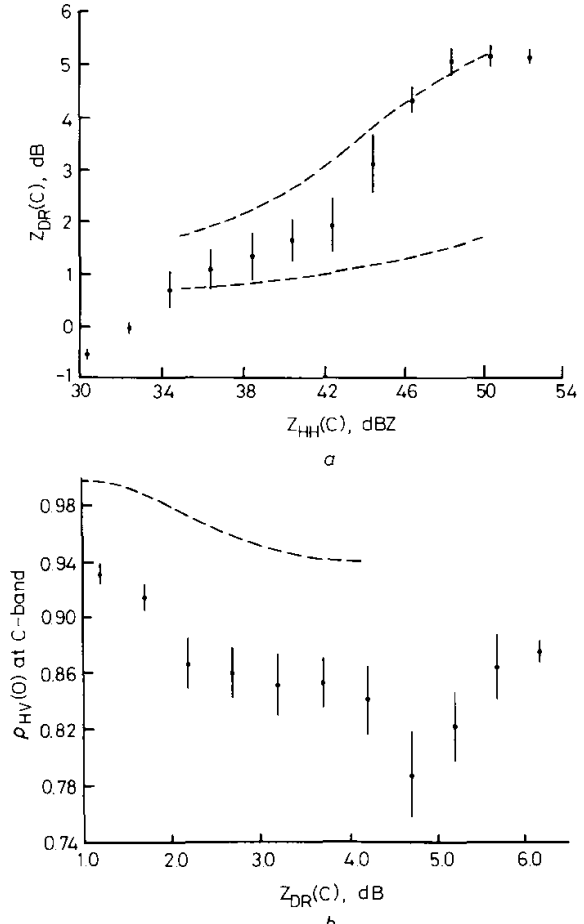

Fig. 14 Averaged radar data based on 20 range profiles similar to Fig. 13 taken in the same convective storm

Vertical lines correspond to the standard deviation

a $Z_{D R}$ dala have been averaged over $3 \mathrm{~dB}$ categories of $Z_{H H}$

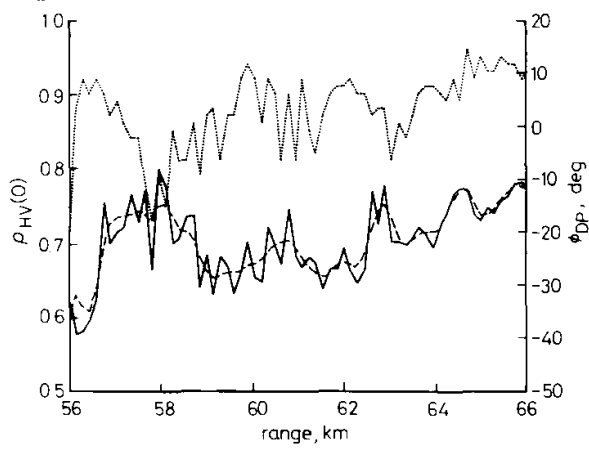


(same azimuth and elevation). The $Z_{D R}$ data have been averaged over $3 \mathrm{~dB}$ categories of $Z_{H H}$. Two distinct $Z_{D R}$ regions may be identified, corresponding to $Z_{H H}<$ $45 \mathrm{dBZ}$ and $Z_{H H}>45 \mathrm{dBZ}$. For $Z_{H H}<45 \mathrm{dBZ}$ the radar data fall within the 'natural' rainfall zone shown in Fig. $1 b$ while for $Z_{H H}>45 \mathrm{dBZ}$, the radar $Z_{D R}$ exceeds the theoretical rain values (at comparable reflectivity levels). Fig. $14 b$ shows $\rho_{H V}(0)$ against $Z_{D R}$ for the 20 ray profiles. The $\rho_{H V}(0)$ data have been averaged over $0.2 \mathrm{~dB}$ categories of $Z_{D R}$. At high $Z_{D R}$, the $\rho_{H V}(0)$ values are significantly smaller than expected for rainfall, see Fig. $3 b$. We interpret the high $Z_{H H}$, high $Z_{D R}$, nonzero $\delta$ and lower $\rho_{H V}(0)$ as being due to rain mixed with small, melting hail.

\section{Summary and conclusions}

Emphasis in this paper has been on presenting experimental data from three radars: the NCAR S-band CP-2 radar, the NSSL S-band Cimarron radar and the DLR $\mathrm{C}$-band Poldirad radar. These radars can measure differential reflectivity $Z_{D R}$, the (zero-lag) crosscorrelation coefficient $\rho_{H V}(0)$, and the propagation differential phase $\left(\phi_{D P}\right)$ between $\mathrm{H}$ and $\mathrm{V}$ polarisation states. The radars were operated in a special time series mode. The transmitted polarisation state was switched between $\mathrm{H}$ and $\mathrm{V}$ states on a pulse-to-pulse basis. The received signal was always copolar to that transmitted. Data in the form of range profiles of reflectivity, $Z_{D R}, \rho_{H V}(0)$ and $\phi_{D P}$ were presented in convective storms. Based on the measurements and a raindrop model, inferences were made regarding the precipitation medium, e.g. rain or rain mixed with ice (hail) within the convective precipitation shaft; or oriented ice crystals mixed with aggregates in the stratiform anvil region of convective storms or mesoscale convective complexes. A raindrop model with gamma raindrop size distributions was used to simulate the radar parameters at $\mathrm{S}$ - and $\mathrm{C}$-bands. The three gamma parameters $\left(N_{o}, D_{o}, m\right)$ were varied over a wide range encompassing a variety of rainfall types. Radar measurements in rainfall were found to be in good, general agreement with the simulations. We also show that $\phi_{D P}$ at $\mathrm{C}$-band need not be monotonic increasing with range, rather the range profile is monotonic increasing with 'bumps' superimposed due to regions of nonzero $\delta$, e.g. regions of large raindrops or small, partially melted ice.

\section{Acknowledgments}

This research was supported by the National Science Foundation (NSF) via grants ATM-8703126 and ATM8915141 and by the US Army Research Office's Center for Geosciences at Colorado State University (CSU). The participation of the CP-2 radar during CINDE was supported by the Center. A co-operative research effort between CSU and DLR was funded by the NSF. Dr. D. Zrnic of the National Severe Storms Laboratory was instrumental in providing us with the opportunity to collect time series data with the Cimarron radar. The Poldirad radar was operated by the Institute of Atmospheric Physics, DLR, Germany. The authors acknowledge the support rendered by Dr. J. Vivekanandan of CSU, and Dr. M. Chandra, Dr. T. Jank and Mr. Fred Ritenberg of DLR. The central roles played by $\mathrm{Mr}$. Grant Gray and Mr. Brian Lewis of NCAR in the CP-2 radar time series collection effort are acknowledged.
7 References

1 VAN ZYL, J.J., PAPAS, C.H., and ELACHI, C.: 'On the optimum polarizations of incoherently reflected waves', IEEE Trans., 1987, AP-35, (7), pp. 818-825

2 OGUCHI T. 'Electromagnetic wave propagation and scattering in rain and other hydrometeors', Proc. IEEE, 1983, 71, pp. 1029-1078

3 MCCORMICK, G.C., HENDRY, A, and BARGE, B.L.: 'The anisotropy of precipitation media', Nature, 1972, 238, (5361), pp. 214-216

4 MCCORMICK, G.C., and HENDRY, A.: 'Principles for the radar determination of the polarization properties of precipitation', Radio Sci., 1975, 10, (4), pp. 421-434

5 MCCORMICK, G.C and HENDRY, A.: 'Techniques for the determination of the polarization properties of precipitation', Radio Sci., 1979,14, pp. $1027-1040$

6 HENDRY, A., ANTAR, Y.M.M., and MCCORMICK, G.C.: 'On the relationship between the degree of preferred orientation in precipitation and dual-polarization radar echo characteristics', Radio Sci., 1987, 22, pp. 37-50. See also correction, ibid., p. 687

7 BEBBINGTON, D.H.O., MCGUINNESS, R.. and HOLT, A.R.: 'Correction of propagation effects in S-band circular polarizationdiversity radars', IEE Proc. H. Microwaves, Antennas \& Propag. 1987, 134, (5), pp. 431-437

8 JAMESON, A.R., and DAVE, J.H.: 'An interpretation of circular polarization measurements affected by propagation differential phase shift', J. Atmos. \& Oceanic Technol., 1988, 5, pp. 405-415

9 MCGUINESS, R., and HOLT, A.: 'The extraction of rain rates from CDR data'. Proc. 24th AMS Conf. on Radar Met., Tallahassee, FL, 27th-31st March 1989, pp. 338-341

10 SELIGA, T.A., and BRINGI, V.N.: 'Potential use of radar differential reflectivity measurements at orthogonal polarizations for measuring precipitation', J. Appl Meteorol, 1976, 15, pp 69-76

11 Suring precipitation', A. Appl. Meteorol.,

HALL, M.P.M., GODDARD, J.W.F., and CHERRY, S.M. 'Identi-
fication of hydrometeors and other targets by dual-polarization fication of hydrometeors and other targe
radar', Radio Sci., 1984, 19, (1), pp. 132-140

12 GIULI, D.: 'Polarization diversity in radar', Proc. IEEE, February $1986,74,(2)$, pp. $245-269$

13 POELMAN, A.J.: 'Virtual polarization adaptation. A method of increasing the detection capability of a radar system throgh polarization-vector processing', IEE Proc. F. Commun. Radar \& polarization-vector processing, $1 E E$
Signal Process. 1981, 128, pp. 261-270

14 POELMAN, A.J., and GUY, F.R.F.: 'Polarization information utilization in primary radar', in BOERNER, W.M. (Ed.): 'Inverse methods in electromagnetic imaging' (D. Reidel Publ. Co., 1985), pp $521-572$

15 BRINGI, V.N., and HENDRY, A.: 'Technology of polarization diversity radars for meteorology' in ATLAS, D. (Ed). 'Radar in meteorology' (American Meteorol. Soc., Boston, 1990), Chap. 19a, pp. $153-190$

16 SP. 153-190 C-band coherent polarimetric radar for propagation and cloud
(a) physics research', J. Atmos. \& Oceanic Technol., 1988, 5, pp. 803-822

17 HENDRY, A., MCCORMICK, A.C., and BARGE, B.L.: 'KU-band and S-band observations of the differential propgation constant in snow', IEEE Trans., 1976, AP-24, (4), pp. 521-525

18 ULBRICH, C.W - "Natural variations in the analytical form of raindrop size distributions', J. Clim. \& Appl. Meteorol., 1983, 22, 1764 1775

19 PRUPPACHER, H.R., and PITTER, R.L.: 'A semi-empirical determination of the shape of cloud and raindrops', J. Atmos. Sci., 1971 , 28, pp. $86-94$

20 GREEN, A.W.: 'An approximation for the shape of large raindrops', J. Appl. Meteorol., 1975, 14, pp. 1578-1583

21 BEARD, K. . and CHUANG, D. 'A new model for the equilibrium shapes of raindrops', J. Atmos. Sci., 1987, 44, pp. 1509-1524

22 BEARD, K.V., and JAMESON, A.R - 'Raindrop canting', J. Atmos. $S_{C i,}$ 1983, 40, pp. 448-454

23 MEISCHER, P., JANK, I., BRINGI, V.N., and VIVEKANAN DAN, J.: 'Multiple plane $Z_{D R}$ measurements in convective and stratiform clouds using the C-band polarimetric DFVLR radar' Preprints 24th AMS Radar Met. Conf., Tallahasse, Florida, March 1989

24 JAMESON, A.R.: 'Microphysical interpretation of multiparameter radar measurements in rain. Part I: 'Interpretation of polarization measurements and estimation of raindrop shapes', $J$. Atmos. Sci. 1983,40 , pp. 1792-1802

25 SACHIDANANDA, M., and ZRNIC, D.S.: 'Differential propagation phase shift and rainfall rate estimation', Radio $S c i ., 1986,21$, pp $235-247$

26 JAMESON, A.R., and MUELLER, E.A.: 'Estimation of propagation differential phase shift from sequential ortogonal linear polarization radar measurements', J. Atmos. \& Oceanic Technol., 1985, 2 , pp. 133-137

IEE PROCEEDINGS-F, Vol. 138, No. 2, APRIL 1991 
27 BALAKRISHNAN, N., and ZRNIC, D.S.: 'Suggested use of crosscorrelation between orthogonally polarized echoes to infer hail size'. Proc. 24th AMS Conf. on Radar Met., 27th-31st March 1989, pp. 292-296

28 BRINGI, V.N., RASMUSSEN, R.M., and VIVEKANANDAN, J.: 'Multiparameter radar measurements in Colorado convective storms. Part I: Graupel melting studies', J. Atmos. Sci., 1986, 43, (22), pp. 2545-2563

29 VIVEKANANDAN, J., BRINGI, V.N., and RAGHAVAN, R. 'Multiparameter radar modelling and observations of melting ice', $J$. Atmos. Sci., 1990, 47, (5), pp. 549-564

30 BALAKRISHNAN, N., and ZRNIC, D.S.: 'Estimation of rain and hail rates in mixed phase precipitation, J. Atmos. Sci., 1990, 47, pp. $565-583$

31 PRUPPACHER, H.R., and KLETT, J.D.: 'Microphysics of clouds and precipitation' (Reidel, 1978)

32 SACHIDANANDA, M., and ZRNIC, D.S.: 'Efficient processing of alternately polarized radar signals', $J$. Atmos. \& Oceanic Technol. $1989,6,(1)$, pp. $173-181$

33 MUELLER, E.A.: 'Calculation procedure for differential propagation phase shift'. Preprints of 22nd AMS Conf. on Radar Meteorology, 10th-13th September 1984, Zurich, Switzerland, pp. 397-399
34 DOVIAK, R.J., and ZRNIC, D.S.: 'Doppler radar and weather observations' (Academic Press, New York, 1984)

35 BRINGI, V.N., SELIGA, T.A., and CHERRY, S.M.: 'Statistical properties of the dual-polarization differential reflectivity $\left(Z_{D R}\right)$ radar signal, IEEE Trans. Geosci. Remote Sensing, 1983, 21, pp. radar signal,
$215-220$

36 SACHIDANANDA, M., and ZRNIC, D.S. ' $Z_{D R}$ measurement consideration for a fast scan capability radar', Radio Sci., 1985, 20, pp. 907-922

37 CHANDRASEKAR, V., BRINGI, V.N., BALAKRISHNAN, N. and ZRNIC, D.S.: 'Error structure of multiparameter radar and surface measurements of rainfall. Part III: Specific differential phase', J. Atmos. \& Oceanic Technol., 1990, 7, (5), pp. 621-629

38 TUTTLE, J.D., BRINGI, V.N., ORVILLE, H.D., and KOPP, F.J.: 'Multiparameter radar study of a microburst: Comparison with model results', J. Atmos. Sci., 1989, 46, (5), pp. 601-620

39 CAYLOR, J.I., and ILLINGWORTH, A.: "Identification of the bright band and hydrometeors using copolar dual polarization radar. Preprints of 24th AMS Conf on Radar Met, 27th 31st March 1989, Tallahassee, Florida, American Meteorological Society, pp. 9-12 